\title{
Norms for modeling agents' interaction in ubiquitous environments
}

\author{
Ismail Khalil Ibrahim \\ Institute of Telecooperation, Johannes Kepler University Linz, Altenberger Str. 69, A-4040 Linz, Austria \\ Tel.: +43 73224689238; Fax: +43 73224689829; E-mail: ismail@tk.uni-linz.ac.at
}

\begin{abstract}
In ubiquitous environments a vast amount of mobile human and software entities, each with limited resources and knowledge, needs to interact with each other to achieve common and/or individual goals within a specific context. Due to their autonomy, proactiveness, mobility, social capability, and the successful implementation of agent mediated applications and services over the Web, different scenarios have been proposed in literature for the use of agents in ubiquitous environments for a wide range of applications such as user interfaces, mobile computing, information retrieval and filtering, smart messaging, telecommunication and m-commerce. In this paper, we address the problem of modeling agents' interaction in ubiquitous environments. We argue agents' interactions can be modeled using norms, which are represented mathematically as zero set of $\mathrm{n}$-variate functions. We utilize the barycentic coordinates to show how agents should behave based on established protocols, rules of conduct or accepted social practices.
\end{abstract}

Keywords: Ubiquitous environment, agents' interaction, norms, electronic institutions

\section{Introduction}

\subsection{Ubiquitous environment}

In social terms, environment [2] is defined as the external elements and conditions that influence the growth, development, and survival of an individual or an organization. It provides the conditions under which individuals and organizations exist. In the same way, we define the ubiquitous environment as the networked infrastructures and technologies, which enable mobile entities (agents, objects, and humans) to communicate anytime, anywhere and by all means for conducting personal, social and business activities. The infrastructure that supports this ubiquitous environment must meet two requirements, first the internal autonomy requirement of participating entities in which interaction and the structure of the environment must be represented independently from the internal design of the participating entities and second the collaboration autonomy requirement in which activities and interaction in the environment must be specified without completely fixing in advance the interaction structure. Due to their autonomy, proactiveness, mobility, social capability, and distributed nature, and the successful implementation of agent mediated application and services over the Web (e-commerce, e-government, e-health, e-learning, etc.) different scenarios have been proposed in the literature for the use of agents in ubiquitous environments for a wide range of applications such as user interfaces, mobile computing, information retrieval and filtering, smart messaging, telecommunication and $\mathrm{m}$-commerce $[1,3,11,14]$. The ultimate goal is to build a mobile, flexible, open, collaborative, sharing, and distributed serviceoriented environment that is able to respond in a timely and ubiquitous manner to user needs and business intelligence. 


\subsection{Modeling agents' interaction in ubiquitous environments}

Agents need an environment to operate and exist [13]. Without the environment, agents are simply useless or never exist. Interaction is the act or process where agents communicate in a way to influence the behavior or belief of each other [6]. Agents' interaction takes the form of coordination, cooperation, collaboration, negotiations, contracts, agreement, communication protocols, and speech acts [13]. Agents' interaction in ubiquitous environment [10] is generally characterized by 1) openness, describing the possibilities that these mobile entities can join or leave the environment, 2) flexibility indicating the degree the mobile entities are restricted by the environment rules and norms, 3) stability defining the predictability of the consequences of actions and 4) trustfulness specifying the extent to which users may trust the environment. Social aspects of multi-agents systems [5,7] have received a lot of attention from the community since social ability is one of the most important attributes of agents which has opened up new modes of interaction and dynamic organizational configurations that were previously inconceivable within a wide array of human and business activities.

The concept of norms can be introduced in ubiquitous environments which is interpreted in different contexts and understood to mean [12] a standard expectation about what behavior, thoughts, or feelings are appropriate within a given group (of agents) within a given context (i.e., location, time, environment).

Most important for us is the definition of norms from agent-based computing perspective [4,7], where a norm is viewed as an expression of the obligations and rights connected to the role an agent has within a larger social system. Norms are descriptions of how an agent should behave in order to comply with the business practices and legal standards. Norms can be created from different sources, varying from built in norms to simple agreements between business parties to more complex legal systems. The above definition classifies norms into explicit norms, context dependent norms and time dependent norms. Dignum [4] set the requirements for the application of norms in distributed artificial intelligence such that norms are always prescribed to be complied with for a set of agents i.e., they specify something that ought to be done and consequently they include normative goals that must be addressed, norms are always applicable and their activation depends on the context in which agents are situated i.e., there maybe situations where agents are not obliged to comply with norms, and norms suggest the existence of a set of sanctions or punishments to be imposed when an agent doesn't satisfy the normative goal, and a set of rewards to be received when agents do.

Two approaches have been proposed in the literature for modeling agents' interaction in ubiquitous environments; norm-aware agents [4,5,11] and electronic institutions [7-9]. In norm aware agents, norms are hard coded into the agent architecture as restrictions resulting in the reduction of the set of possible options, at the decision level or the protocol level, agents can follow to define the ways of reacting to messages ensure coordination [8]. This approach results in according to [5] loss of autonomy since agents always follow strictly the protocol they use to communicate messages and react to messages in a predefined way which may lead also to gain control over the agent, and loss of adaptability since fixed protocols do not have the capacity to react to changes in the environment. In the electronic institution approach $[4,8]$, agents' interactions are regulated by norms, which are represented explicitly to influence agents behavior once they decide to participate in the electronic institution. The concept of electronic institutions was first introduced by [8] to refer to the agents' counterpart of human organizations. They constitute the framework for enabling, through communication, interaction between agents using a set of explicit institutional norms and rules. They are specifically designed for providing support, trust and legitimacy in agent based applications through a set of tools and services for implementing and regulating agents' interactions. 
The approach of electronic institutions raises two problems. The first problem is the implementation of norms in electronic institutions. Norms are usually expressed in abstract, vague manner in the form of what is right (legal) and what is wrong (illegal) in the society without adhering a concrete specification of what is the meaning of right and wrong from a software engineering point of view. In [9], we proposed three levels of abstractions for norms in electronic institutions; 1) values as the embodiment of what an electronic institution stands for, and should be the basis for the behavior of its members, 2) normative behavioral rules which are the rules of conduct within the electronic institution, and 3) low level norms which is the lowest level of abstraction. Examples are social norms (organization, roles, authority), communication norms (protocols, dialogues), and behavioral norms (obligations, permissions, interdiction). The second problem with this approach is the need for institutional agents that are used by the electronic institution to control, monitor and verify that all the agents in the electronic institution are complying with the set of norms specified by the system. In [10], we proposed an algorithm for norms enforcement as a cooperation strategy in electronic institutions by introducing substitution rules that map the norms set for the electronic institution as values to normative rules that can be implemented in the protocols to govern agents' interactions. In addition to centralization of control, reducing the autonomy of agents, liability is another issue.

\subsection{Norms for agents interaction}

The ubiquitous environment defines the collaborative, shared and open properties of the world in which agents, humans, objects, applications, and services utilize the infrastructure and technology to interact with each other. One can identify three components for such an environment:

- The application environment which defines the type of software applications, methods and operations that can run in the environment and the resources needed for running such applications,

- the service environment, which defines the types and communication interfaces of the available services, service description and publishing, services discovery mechanisms, looking up and joining services, and services binding and maintenance once they are discovered, and

- the network environment which defines the network infrastructure, configuration, topology, QoS provision, and the degree of mobility of users and devices like user location, handover, notification and addressing mechanisms.

Without sacrificing the richness of our approach, the environment is solely modeled as a function of agents that exist in the environment and interact with each other by the exchange of speech acts, roles as a standardized pattern of behavior which defines what rights, duties, and opportunities an agent adopting a specific role can have, services (type, ontology, description language, communication protocol, etc.), resources (devices capabilities, bandwidth, etc.), norms (universal laws or restrictions that define the reaction of the environment to agent actions) and context (as a function of time, location, and activities the user is involved in and defined in the user profile implemented on the mobile device). For such environment, we use different types of agents for managing the process of creating, adapting, regulating, maintaining and terminating services according to a predefined set of regulations, rules, and policies that can be used for network management and organization, tasks distribution, resource sharing, admission control, QoS specification and negotiation, etc. These policies are represented as a set of norms describing obligations; permissions and prohibitions, which indicate the type of behavior each of the agents, should adhere to within the environment.

In this paper, we concentrate on the use of these norms in order to model agent interaction. Therefore, it is beyond the scope of this paper to discuss the details on how these abstract, declarative concepts 
can be translated into protocols and procedures that can be implemented from a software point of view. Readers who are interested in this can refer to our pervious work published in $[9,10]$.

For this purpose, we define two types of norms:

\section{Explicit Norms}

These are the simplest form of norms, which can be modeled using deontic modalities expressed as obligations, prohibitions and permissions. Examples of such type of norms are those related to organizational (roles, authority, admission control, security, privacy, etc.), communication (protocols, dialogues), and behavioral (do this, don't do that, do this because everybody is doing this, etc.) aspects of agents' interactions. These norms are active all the time and under all circumstances.

\section{Contextual Norms}

Context is any information that can be used, when correctly interpreted, to characterize the situation of entities (whether a person, place, or object), which are considered relevant to the interaction between a user and a service. Contextual norms are those norms that are holding only with respect to a specific context. Three levels of contextual norms can be identified; location-dependents norms, which are active based on the physical or virtual location of the user, time-dependent norms, which are active only at a particular time slot, and activity-dependent norms which are active only under particular condition in which activities are happening or being done.

\section{Modeling agent interaction using norms}

Agent based computing opens up new opportunities for creating distributed, intelligent, integrated and cooperative mobile services that make use of contextual information about the user and the environment in order to provide the user with the right service, at the right time, and in the right place.

Service provision in ubiquitous environments represents a major challenge. In wired networks, several techniques have been proposed (e.g., XML, SOAP, WSDL, UDDI, Jini, SLP, etc.) for service description and publishing, service finding and discovery, and service binding, which cannot be applied in ubiquitous environments because of the lack of a kind of centralized directory servers like in wired networks due to the fact that all devices, hosts in ubiquitous environment are mobile and have very limited capabilities (computing power, energy constraints, memory, etc.) that make it infeasible for them to act as directory servers. Another problem with ubiquitous environments is, due to bandwidth constraints and dynamic topology of mobile networks, supporting Quality of Service (QoS) for example becomes a challenging task. To support QoS, the link information such as delay, bandwidth, cost, loss rate, and error rate should be available which is difficult to manage. Instead an agent based system for QoS provision and management can be proposed where QoS parameters are set as norms expressing permissions, obligations and other more complex normative relations that regulates the behavior and interaction of agents representing service providers, service brokers, and service requesters and used to guarantee a certain level of QoS. Examples of these rules are those related to QoS models, resource sharing, QoS routing, QoS MAC, self-organization, task orientation which combine the communication properties, resources and location of agents.

\subsection{Modeling norms}

Explicit norms can be modeled using algebraic equalities i.e., using a zero set of n-variate function. $n$-variate functions are used because 1) $\mathrm{n}$-variate function is a function of $\mathrm{n}$ variables which can be used 


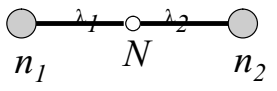

a) 2 norms

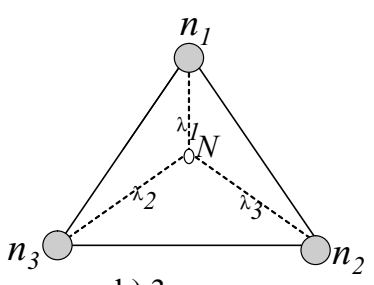

b) 3 norms

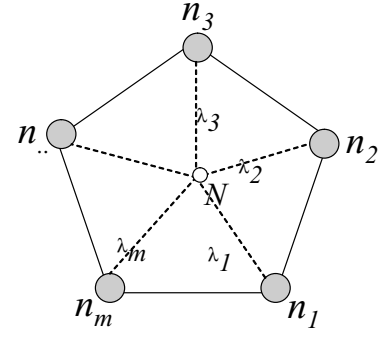

c) $m$ norms

Fig. 1. Norms combination.

to represent different parameters of QoS, 2) The zero set of a function $f\left(x_{1}, x_{2}, \ldots, x_{n}\right)$ is the set $\{($ $\left.\left.x_{1}, x_{2}, \ldots, x_{n}\right) \mid f\left(x_{1}, x_{2}, \ldots, x_{n}\right)=0\right\}$ and 3 ) Any function can be restricted to a subset of its domain. The restriction of $f: A \diamond B$ to $S$, where $S \subseteq A$ or $f \mid S: S \rightarrow B$.

As an example, the norm $N$ can be modeled as the zero set of the bi-variate function $f\left(x_{1}, x_{2}\right)=x_{1}^{2}+x_{2}^{2}$ $-n^{2}$ which represent the set of all points that belong to the norm $n$ such that $f\left(x^{1^{\prime}}, x^{2^{\prime}}\right)=0$ for all $n$ $\left(x^{1^{\prime}}, x^{2^{\prime}}\right)$ that belongs to $n$.

More than one norm can be modeled separately using a zero set of n-variate function for each norm and the zero sets can be combined together. In the case of two norms, we define the overall norm $N$ that can be modeled as a linear combination of $n_{1}$ and $n_{2}$, where and $\lambda_{2}$ are the mathematical ratio of the contribution of $n_{1}$ and $n_{2}$.

$$
N=\frac{\lambda_{2} n_{1}+\lambda_{1} n_{2}}{\lambda_{1}+\lambda_{2}}
$$

In the case of more than two norms, the overall norm $N$ can be modeled as follows: The norms $n_{1}, n_{2}$, $\ldots, n_{m}$ can be seen as vertices of a convex polygon, see Fig. 1 .

The overall norm $N$ is represented by the barycentric coordinates of a point $p$ located inside the polygon. Let $\Omega$ be a convex polygon in the plane, regarded as a closed set, with vertices (modeling the norms) $n_{1}, n_{2}, \ldots n_{m}$ where $m \geqslant 3$.

We call any set of functions $\lambda_{1}: \Omega \longrightarrow \Re ; i=1, \ldots, m$, where $\Re$ is the set of real numbers, barycentric coordinates if they satisfy, for all $v \in \Omega$, the following three properties:

$$
\begin{aligned}
& \lambda_{i}(v) \geqslant 0 ; \quad i=1, \ldots, m \\
& \sum_{i=1}^{m} \lambda_{i}(v)=1 \\
& \sum_{i=1}^{m} \lambda_{i}(v) v_{i}=v
\end{aligned}
$$

This definition does indeed generalize triangular barycentric coordinates, in the case of $n=3, \Omega$ is the triangle. The contribution ratio, $\lambda_{2}, \ldots$ can be calculated as the areas of the sub-domains $A_{1}, A_{2}, A_{3}$. 
And the properties (3) and (4) alone determine the three coordinates uniquely, namely

$$
\lambda_{1}(n)=\frac{N\left(n, n_{2}, n_{3}\right)}{N\left(n_{1}, n_{2}, n_{3}\right)}, \quad \lambda_{2}(n)=\frac{N\left(n_{1}, n, n_{3}\right)}{N\left(n_{1}, n_{2}, n_{3}\right)}, \quad \lambda_{3}(n)=\frac{N\left(n_{1}, n_{2}, n_{)}\right.}{N\left(n_{1}, n_{2}, n_{3}\right)}
$$

where $N\left(n_{1}, n_{2}, n_{3}\right)$ denotes the signed area of the triangle $\left[n_{1}, n_{2}, n_{3}\right]$.

For example, with $n=(x, y)$ and $v_{i}=\left(x_{i}, y_{i}\right)$, we have $i=2,3$.

$$
N\left(v, v_{2}, v_{3}\right)=\frac{1}{2}\left|\begin{array}{lll}
1 & 1 & 1 \\
x & x_{2} & x_{3} \\
y & y_{2} & y_{3}
\end{array}\right|
$$

The functions $\lambda_{1}, \lambda_{2}, \lambda_{3}: \Omega \longrightarrow \Re$ are thus linear polynomials and non-negative.

\subsection{Application}

Different types of explicit and contextual norms at different levels of granularity can be defined with regard to QoS provision and management in wireless networks namely, accuracy, perception, timeliness, price and convenience defined at the user layer, frame rate, frame size, response time, throughput and security defined at the application level, buffer size, process priority, scheduling, policy, caching policy, and time quantum at the system level, or bandwidth, throughput, bit-error rate, end-to-end delay, delay jitter, and peak durations defined at the network level.

If we define norm space as the domain of all entries, which satisfy the overall norm (modeled as function $\mathrm{N}$ ), i.e. all the elements of the zero set of $\mathrm{N}$, then the context space is the sub-domain where the related barycentic coordinates area is defined. Clearly the context sub-domain is the key factor in determining the related contribution mathematical ratio $\lambda$.

Let us assume that an agent is restricted to three norms; the first one is related to the response time (we call it $n_{t}$ ), the second is related to the available bandwidth (we call it $n_{b}$ ) and the third is related to the end-end delay (we call it $n_{d}$ ). We want to maintain a quality of service that make the agent satisfy these three norms. Let us model these norms as the vertices of a triangle $T B D$, where $T$ stand for response time, $B$ stands for bandwidth and finally $D$ stands for delay. The overall norm $N$ is modeled as

$$
N=\frac{n_{d} \lambda_{t} \lambda_{b}+n_{b} \lambda_{b} \lambda_{d}+n_{b} \lambda_{t} \lambda_{d}}{\lambda_{t}+\lambda_{b}+\lambda_{d}}
$$

The norm space, triangle $T B D$, is bounding the domain of the QoS, moving inside the norm space, i.e. the value of the contribution ratios $\lambda_{1}, \lambda_{2}, \lambda_{3}$ are modified according to how far the agent is from the norms $n_{1}, n_{2}, n_{3}$. The QoS parameters decide the size of the domains $A_{1}, A_{2}, A_{3}$ and accordingly the overall norm. Based on priority of the QoS, one can introduce the concept of assigning weights to highly priority norms. In the case of $m \geqslant 3$, the weighted barycentric coordinates can be used. Introducing weights provide us the possibility to set up the priorities according to the user preferences, or performance characteristics such as delay, jitter, bit error rate, packet lose and more. In this case, the overall function can be modeled as:

$$
N=\frac{w_{1} \lambda_{2} n_{1}+w_{2} \lambda_{1} n_{2}}{\lambda_{1}+\lambda_{2}}
$$

where $w_{1}$ and $w_{2}$ are priorities assigned by the user to different QoS parameters. This depicted in Fig. 2 where priority is given to $n_{2}$ over the other two norms. 


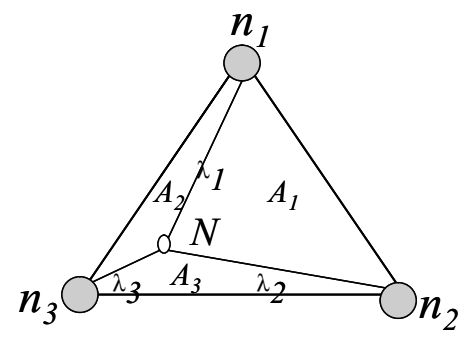

Fig. 2. Weighted norms.

\section{Conclusions}

Agents' properties like autonomy, proactiveness, social ability, etc., make them a promising tool to be used in ubiquitous environments where small devices with limited capabilities need to interact with each other and with users to carry on personal, social and business activities. This brings up issues like networks management and organization, resource sharing, tasks distribution, admission control, QoS specification and negotiation, etc., which limits the flexible and adaptive deployment of agents for useful applications and services. In this paper, we have addressed the problem of modeling agents' interaction in ubiquitous environments using a set of norms. Norms are modeled as zero set of n-variate functions. We utilize barycentic coordinates to show how agents should behave based on established protocols, rules of conduct or accepted social practices. We applied this approach to QoS management where agents behavior is controlled by QoS parameters in order to ensure a guaranteed QoS in wireless networks.

\section{References}

[1] C. Carabelea and O. Boissier, Multi-agent platforms on smart devices: Dream or reality? in Proceedings of the Smart Objects Conference (SOC03), Grenoble, France, 2003, 126-129.

[2] E. Chang, T.S. Dillon and F.K. Hussain, Trust and Reputation for Service-Oriented Environments, John Wiley and Sons, UK (400 pages), 2006, ISBN: 0-470-01547-0.

[3] S.A. DeLoach and J.L. Valenzuela, An Agent-Environment Interaction Model, in: AOSE 2006, LNCS 4405, L. Padgham and F. Zambonelli, eds, Springer-Verlag, Berlin Heidelberg, 2007, pp. xx-xx.

[4] F. Dignum, Abstract norms and electronic institutions. In Proceedings of the International Workshop on Regulated Agent-Based Social Systems: Theories and Applications (RASTA '02), Bologna, 2002, 93-104.

[5] V. Dignum, A Model for Organizational Interaction: Based on Agents, Founded in Logic, Ph.D. thesis, Universiteit Utrecht, The Netherlands, 2004.

[6] V. Dignum, J.-J. Ch. Meyer, F. Dignum and H. Weigand, Formal specification of interaction in agent societies. In 2nd Goddard Workshop on Formal Approaches to Agent-Based Systems (FAABS). Maryland, 2002b.

[7] M. Esteva, Electronic Institutions: from specification to development. Ph.D. thesis, Universitat Politechnica de Catalunya, 2003.

[8] M. Esteva, J. Padget and C. Sierra, Formalizing a language for institutions and norms, in: Intelligent Agents VIII, LNAI 2333, J.-J. Ch. Meyer and M. Tambe, eds, Springer Verlag, 2001, 348-366.

[9] K. Ibrahim, G. Kotsis and W. Schwinger, Mapping Abstractions of Norms in Electronic Institutions, Proceedings of the 12th IEEE International Workshops on Enabling Technologies: Infrastructure for Collaborative Enterprises (WETICE2003), June 9-11, 2003, Linz, Austria, 30-35.

[10] K. Ibrahim, R. Kronsteiner and G. Kotsis, Norms Enforcement as a Coordination Strategy in Ubiquitous Environments, in Embedded and Ubiquitous Computing: EUC 2005 Workshops: UISW, NCUS, SecUbiq, USN, and TAUES, Nagasaki, Japan, December 6-9, 2005, Springer LNCS 3823/2005, ISBN: 3-540-30803-2, 355-364.

[11] L.D. Kamara, J. Pitt and M. Sergot, Norm aware agents for Ad Hoc networks, Proceedings AAMAS Workshop on Agents and Ubiquitous Computing, 20 July 2004, New York, 2004, 1-8.

[12] J.-J. Ch. Meyer and R.J. Wieringa, eds, Deontic Logic in Computer Science. Wiley Professional Computing, England, 1993. 
[13] J. Odell, H.V.D. Parunak, M. Fleischer and S. Brueckner, Modeling Agents and their Environment, in: Agent-Oriented Software Engineering III: Third International Workshop, F. Giunchiglia, J. Odell, and G. Weiss, eds, AOSE 2002.

[14] K. Trzec and D. Huljenic, Intelligent agents for QoS management, Proceedings of the first international joint conference on Autonomous agents and multi-agent systems, 2002, 1405-1412.

Dr. Ismail Khalil Ibrahim is a Senior Researcher and Lecturer at the Institute of Telecooperation- Johannes Kepler University Linz, Austria, where he teaches, consults, and conducts research in mobile multimedia applications and services, agent technologies, and information integration. He received his M.Sc. and Ph.D. in Computer Engineering and Information Systems from Gadja Mada University, Indonesia. Dr. Ibrahim previously served as a Research Fellow at Intelligent Systems Group in the Netherlands. He is Editor in Chief of the International Journal of Web Information Systems (JWIS), the Journal of Mobile Multimedia (JMM), the Journal of Mobile Computing and Multimedia Communication. His research interests also include business, social, and policy implications associated with the emerging Web technologies. 

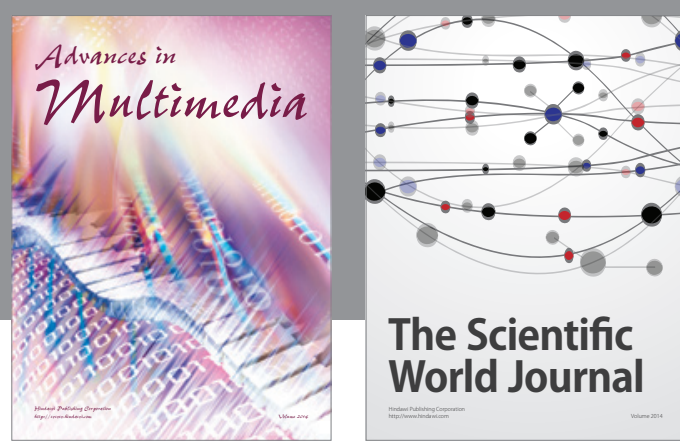

The Scientific World Journal
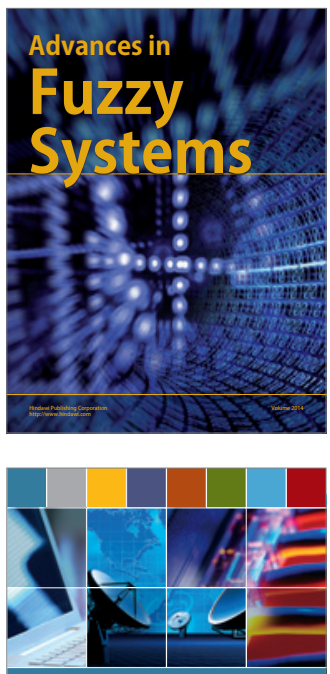

Computer Networks and Communications
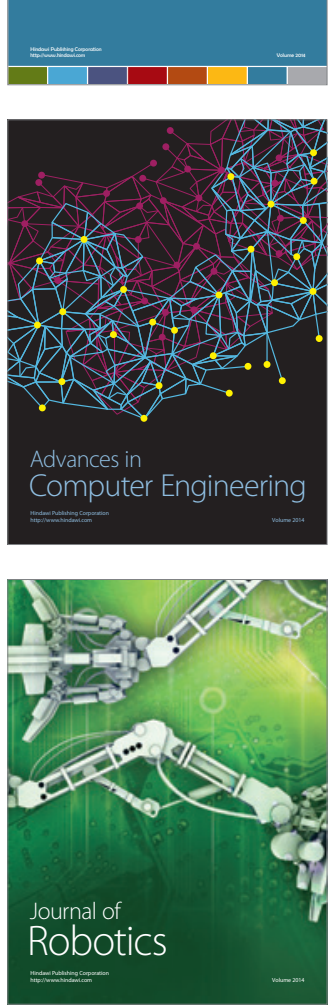
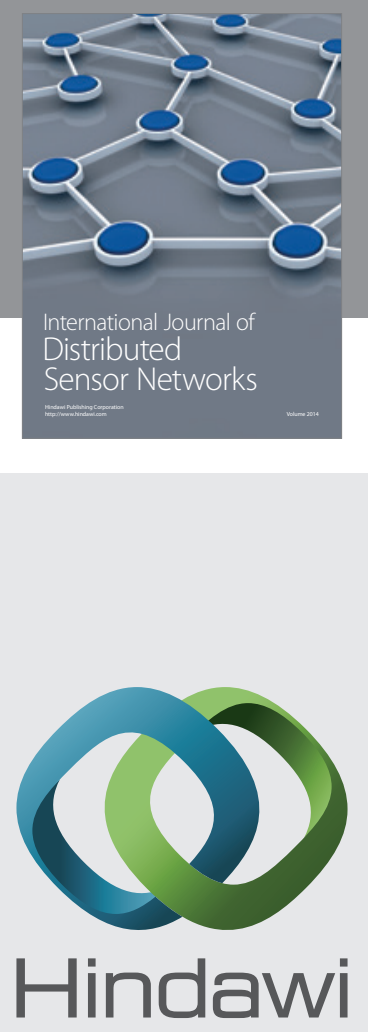

Submit your manuscripts at

http://www.hindawi.com
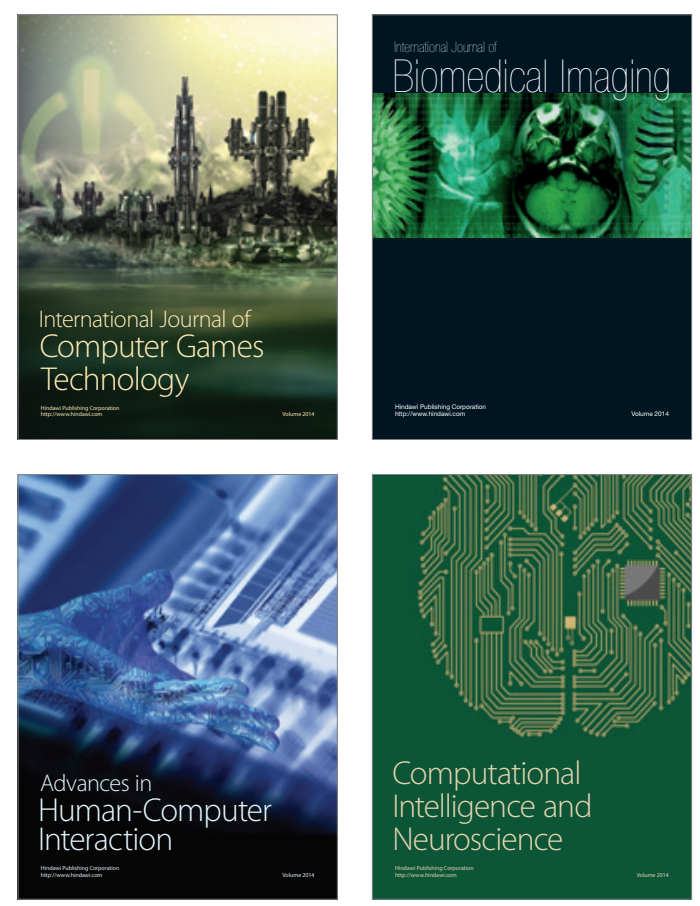
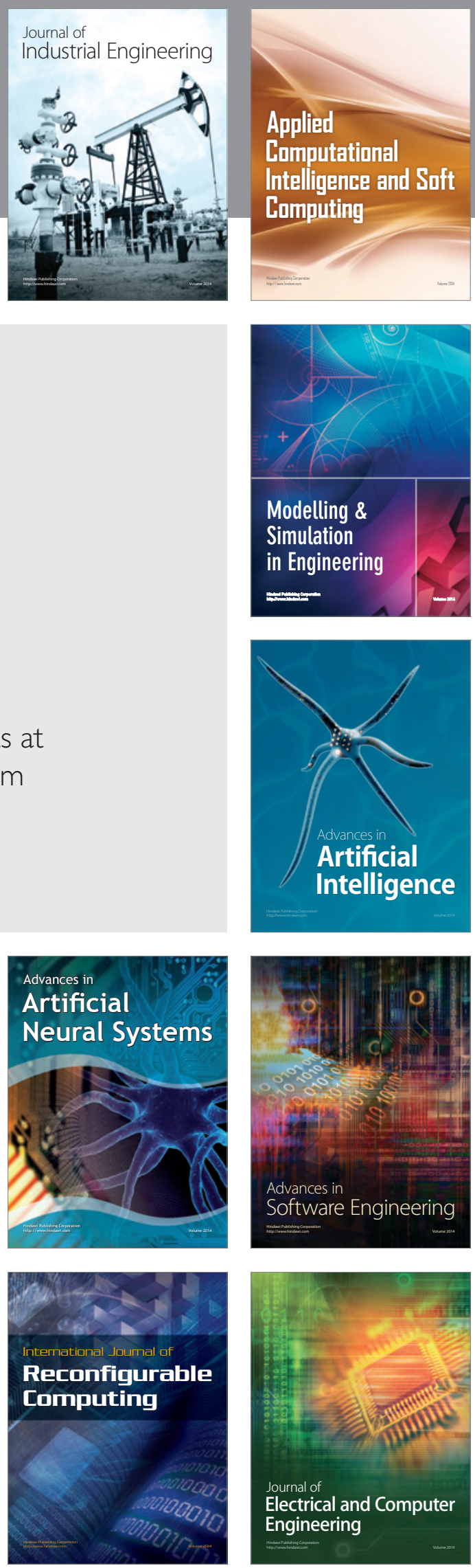